\title{
Exercise Therapy for an Older Patient With Left Ventricular Assist Device
}

\author{
Won Hah Park, $\mathrm{MD}^{1}$, Yong Gon Seo, $\mathrm{MS}^{1}$, Ji Dong Sung, $\mathrm{MD}^{2}$ \\ ${ }^{1}$ Divison of Sports Medicine, Department of Physical Medicine and Rehabilitation, \\ ${ }^{2}$ Department of Internal Medicine, Samsung Medical Center, Sungkyunkwan University School of Medicine, Seoul, Korea
}

\begin{abstract}
A left ventricular assist device (LVAD) is a mechanical circulation support implanted for patients with end-stage heart failure. It may be used either as a bridge to cardiac transplantation or as a destination therapy. The health of a 75-year-old man with a medical history of systolic heart failure worsened. Therefore, he was recommended to have implanted a LVAD (Thoratec Corp.) as a destination therapy. After the surgery, he was enrolled in patient cardiac rehabilitation for the improvement of dyspnea and exercise capacity. In results, there is an improvement on his exercise capacity and quality of life. For the first time in Korea, we reported a benefit of exercise therapy after being implanted with a LVAD.
\end{abstract}

Keywords Left ventricular assist device (LVAD), Exercise therapy, Destination therapy

\section{INTRODUCTION}

Chronic heart failure (CHF) is a final stage in progressive development of cardiovascular disease. It affects an estimated 5.1 million Americans, and almost 274,601 people died from heart failure in the United States in 2009 [1]. Patients with end-stage heart failure become severely disabled because of dyspnea at rest or with minimal exertion, despite maximal medical therapy [2]. There are several available treatment options for these patients: medical management, heart transplantation, mechanical

Received March 4, 2013; Accepted August 16, 2013

Corresponding author: Yong Gon Seo

Division of Sports Medicine, Department of Physical Medicine and Rehabilitation, Samsung Medical Center, Sungkyunkwan University School of Medicine, 81 Irwon-ro, Gangnam-gu, Seoul 135-710, Korea Tel: +82-2-3410-3847, Fax: +82-2-3410-6689, E-mail: yongon79@naver. com

(c) This is an open-access article distributed under the terms of the Creative Commons Attribution Non-Commercial License (http://creativecommons. org/licenses/by-nc/3.0) which permits unrestricted noncommercial use, distribution, and reproduction in any medium, provided the original work is properly cited.

Copyright $\odot 2013$ by Korean Academy of Rehabilitation Medicine circulatory support, and hospice care. A left ventricular assist device (LVAD) is a mechanical circulatory support implanted in patients with end-stage heart failure. It may be used either for a short period before cardiac transplantation as a bridging therapy or for a long-term period as a destination therapy for patients who are too sick to undergo cardiac transplantation [2]. Destination therapy for patients who are not candidates for a cardiac transplant is an important treatment for their quality of life (QOL) and survival benefit. In general, patients with an implanted LVAD are transferred to a cardiac rehabilitation facility for early recovery and to prevent complication after cardiac surgery. Although an LVAD implant improves cardiac hemodynamics, it does not improve functional ability, such as ADL and cardiopulmonary function. Rehabilitation for these patients is therefore important and challenging [2]. The purpose of this report is to present our experience with a patient who underwent LVAD implantation, which was performed as a destination therapy for the first time in our country, and then was enrolled for in-patient cardiac rehabilitation. 


\section{CASE REPORT}

A 75-year-old man with systolic heart failure was admitted. He had had an aortic valve replacement due to severe aortic regurgitation in 2000 and had atrial flutter/ fibrillation. The patient was treated with all medical options including medication and intervention therapy (Table 1). Despite the intensive therapy, low left ventricular function persisted with more severe dyspnea. Therefore, the patient was admitted to our hospital in anticipation of mechanical circulatory support. The decision was made to implant a LVAD (Fig. 1). After comprehensive evaluation, he underwent LVAD implant on August 17, 2012. After the surgery, he was transferred to the intensive care unit (ICU) for the care of the surgery site and recovery. Inpatient cardiac rehabilitation was initiated in the ICU on the postoperative day (POD) 3. The first exercise for the patient was a breath technique to prevent pulmonary complication. It included pursed lip breathing and diaphragm breathing, followed by range of motion exercises. During days 3 to 10 at the ICU, the patient had improved with activities of daily living and exercise intensity that were checked using the rating of perceived exertion (RPE). Next day, he was transferred to the ward. During exercise at the ward, we checked perceived exertion using the modified Borg scales to know the level of dyspnea. The mean arterial blood pressure was measured by a hand-held Doppler device (Hadeco Inc., Kawasaki, Japan). The heart rate (HR) and electrocardiogram were checked with a Q-Tel telemetry device (Quinton Instrument Co, Bothell, WA, USA) to monitor the patient's status. We also checked oxygen saturation with an OxiMax N-560 pulse oximeter (Covidien, Boulder, CO, USA). POD 19 , he attempted to ride a stationary bike for 4 minutes 36 seconds (level 0, 50 to $60 \mathrm{rpm}$ ) in our sports medicine center. The target HR was calculated based on the resting

Table 1. Baseline clinical data of the patient

\begin{tabular}{llll}
\hline \multicolumn{1}{c}{ Characteristic } & \multicolumn{2}{c}{ Value } \\
\hline Age & 75 & \\
\hline Sex & Male & \\
\hline Height $(\mathrm{cm})$ & 166.6 & 50 & \\
\hline Weight $(\mathrm{kg})$ & 21.3 & \\
\hline BMI $\left(\mathrm{kg} / \mathrm{m}^{2}\right)$ & 20 & Furosemide $40 \mathrm{mg}$ \\
\hline LVEF $(\%)$ & Systolic heart failure & Cilostazol $50 \mathrm{mg}$ \\
\hline Diagnosis & II & Colchicine $0.6 \mathrm{mg}$ \\
\hline NYHA class & $93 / 57$ & Enoxaparin $60 \mathrm{mg}$ \\
\hline Blood press $(\mathrm{mmHg})$ & 99 & \\
\hline Heart rate $(\mathrm{bpm})$ & Spironolactone $25 \mathrm{mg}$ & \\
\hline Medication & Amiodarone 200 mg & \\
\hline & Isosorbide mono 20 mg & \\
\hline Echo $(2012 / 08 / 16)$ & Loperamide oxide & \\
\hline & S/P aortic valve replacement in 2000 & \\
\hline & Markedly LV dilation with severe LV systolic dysfunction \\
\hline & Moderate tricuspid regurgitation & \\
\hline & Mild mitral regurgitation & \\
\hline & Both atrial enlargement & \\
\hline & Dilated aortic root (43 mm) \& ascending aorta (39 mm) \\
\hline & Borderline pulmonary hypertension & \\
\hline & Minimal amount of pericardial effusion & \\
\hline & Visible redundant material at anterior mitral valve leaflet \\
\hline
\end{tabular}

BMI, body mass index; LVEF, left ventricular ejection fraction; NYHA, New York Heart Association; LV, left ventricular. 
HR plus $30 \mathrm{bpm}$, which was recommended by the American College of Sports Medicine (ACSM) guidelines for exercise testing and prescription. His HR increased (range, 79 to $110 \mathrm{bpm}$ ) during the aerobic exercise. On day 27, he was able to go up and down the stairs of the ward, and he could walk on the treadmill for 10 minutes at a very slow speed (speed of $1.2 \mathrm{~km} / \mathrm{hr}$, grade 0 ) on POD 90. And then, the exercise intensity increased progressively to adjust the RPE and modified Borg scale. The patient was able to walk for 20 minutes at a speed of $2.7 \mathrm{~km} / \mathrm{hr}$, grade 2 (Table 2). Along the course of aerobic exercise training, the level of dyspnea improved relative to the patient's previous condition. His ambulation improved from $80 \mathrm{~m}$ with a walker with supervision to $320 \mathrm{~m}$ independently. We performed a 6-minute walk test (6-MWT), which is a modality available to evaluate functional exercise capacity, in a 15-m unobstructed corridor at post 2,12 , and 20

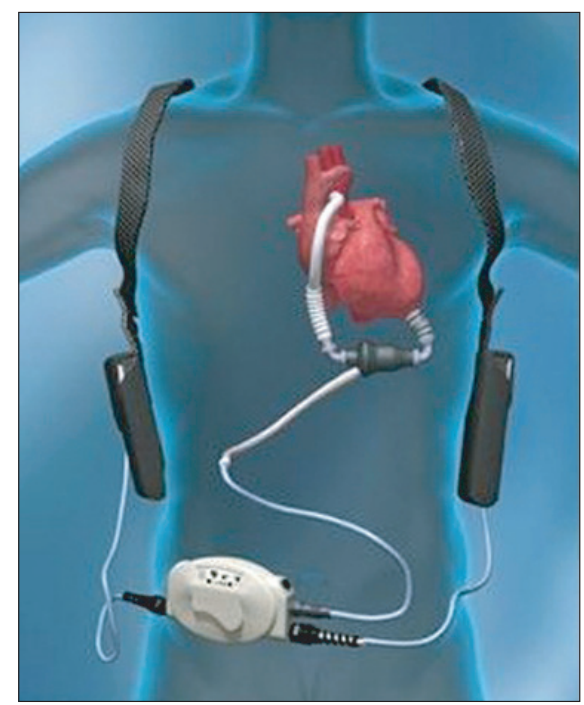

Fig. 1. Components of the HeartMate II continuous-flow left ventricular assist device system, including pump, driveline, system controller, and batteries. weeks. The results of the test were 121, 192, and $285 \mathrm{~m}$, respectively, indicating an improved exercise capacity (Fig. 2). QOL was evaluated with a special questionnaire, Minnesota Living with Heart Failure Questionnaire (MLHFQ). All of his conditions improved on the 4th month relative to POD, and the results of the score decreased (the emotional scores were 23,18 , and 12 , respectively). The patient had some complications after implantation including delayed wound healing, severe dyspnea and non-sustained ventricular tachycardia (NSVT). These factors delayed discharge. He stayed in our hospital for a long period of time and was discharged on POD 136.

\section{DISCUSSION}

This present study is to show the effect of exercise therapy for a patient with an implanted LVAD that is a continuous-flow rotary pump with an axial design. The

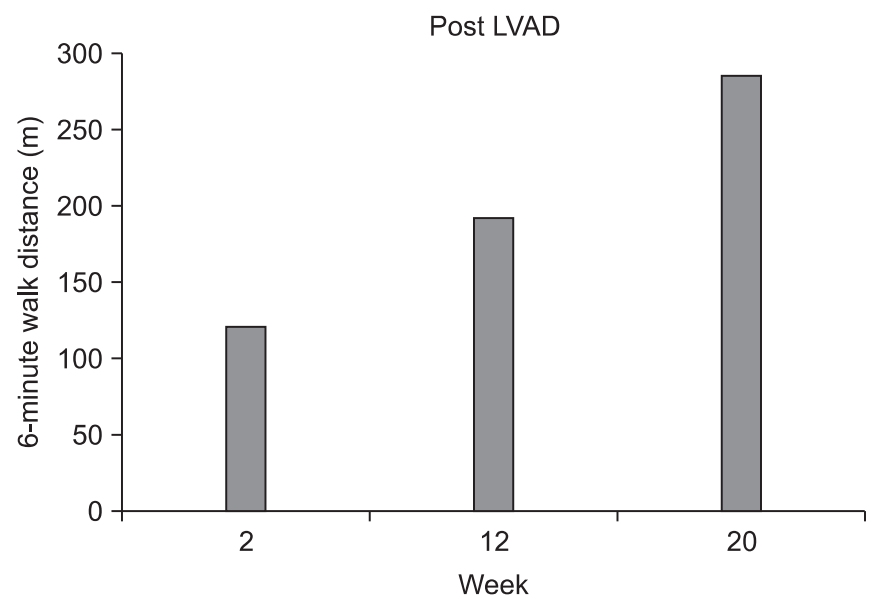

Fig. 2. The 6-minute walk test (6-MWT) results for the patient in this case (bars). There was improvement during left ventricular assist device (LAVD) support from postoperative 2 to 20 weeks.

Table 2. Exercise data obtained from the exercise therapy

\begin{tabular}{lcccccc}
\hline \multicolumn{1}{c}{ Type } & Time (min) & MHR & MBP & MRPE & ASpO $_{2}$ & Modified Borg scale \\
\hline Bed exercise & 30 & 90 & 85 & 13 & 96 & $3-5$ \\
Corridor walking & 20 & 95 & 96 & 13 & 98 & $3-5$ \\
Stairs up and down & 10 & 100 & 98 & 13 & 94 & $3-5$ \\
Bike & 15 & 104 & 100 & 15 & 93 & $3-5$ \\
Treadmill & 20 & 110 & 104 & 15 & 92 & $3-5$ \\
\hline
\end{tabular}

MHR, maximal heart rate; MBP, maximal blood pressure; MRPE, maximal rating of perceived exertion; $\mathrm{ASpO}_{2}$, average saturation of pulse oximetry oxygen. 
Table 3. Score of the MLHFQ dimensions

\begin{tabular}{lccc}
\hline \multirow{2}{*}{\multicolumn{1}{c}{ MLHFQ }} & \multicolumn{3}{c}{ Score } \\
\cline { 2 - 4 } & 4-wk & 12-wk & 20-wk \\
\hline Physical dimension (0-40) & 34 & 26 & 21 \\
\hline Emotional dimension (0-25) & 23 & 18 & 12 \\
Overall score (0-105) & 95 & 77 & 58 \\
\hline
\end{tabular}

MLHFQ, Minnesota Living with Heart Failure Questionnaire.

pump has an operating RPM range of 6,000 to 15,000 and can generate up to $10 \mathrm{~L} / \mathrm{min}$ of flow at an approximate pressure of $100 \mathrm{mmHg}$ [3]. Not only does the exercise intensity progressively increase, but the HR response also increases. In our case, the patient was implanted with a LVAD for destination therapy. After the surgery, the patients with implanted LVAD participated in cardiac rehabilitation to improve general condition. Previous studies show that an intensive exercise therapy after implanted VAD improves exercise capacity in these types of patients [4]. Both early progressive mobilization and exercise training may improve the overall condition and survival rate of VAD patients $[4,5]$. The patient of this study also enrolled in our inpatient cardiac rehabilitation at POD 3. Our exercise therapy program began early in the ICU and then lasted until discharge from our hospital. The main components of exercise therapy were aerobic exercises that enhance cardiopulmonary function. To evaluate cardiopulmonary capacity, the 6 -MWT was carried out in a corridor. The distance walked in meters in a postoperative 6-MWT was the strongest predictor of late post-LVAD mortality [6]. QOL is an important factor to evaluate the prediction of post-surgery condition in LVAD implantation. The MLHFQ was used to evaluate the QOL of patients with CHF. Lower scores indicate better QOL and higher scores indicate the opposite. Therefore, the present study also evaluated QOL using MLHFQ and showed that it was improved at discharge time (Table 3). Daily activities and interaction with other people are restricted in patients with severe heart failure not just because of the physical illness but also because of psychological problems. Also, in our case, being tethered to a lifesustaining device was a major psychological burden. Good family support and participation throughout the recovery process is known to bring a positive effect and to ameliorate mood changes [7]. In the present study, the patient performed walking in the hospital ward as well as aerobic exercises on a stationary bike and on a treadmill to improve breathing, exercise capacity, and functional ability. Exercise intensity increased progressively up to the target HR (upper limit range, $110 \mathrm{bpm}$ ), but stopped exercising when he was not able to continue to the prescribed intensity due to dyspnea. Monitoring of exercise intensity with HR is usually accepted for exercise therapy; however, monitoring with RPE should be used when the HR response is in an unstable state for the exercise or when the patient has taken a medication that affects the HR response. Postoperative medical complications in patients with an LVAD may include bleeding, arrhythmias, infections, device failure, and depression. These complications are likely to affect the patient's length of stay in the hospital. The patient in our case was expected to be discharged from the hospital at POD 14 . However, delayed wound healing may have resulted in a longer stay in the hospital. After POD 30, he had shortness of breath with light physical activity and was getting worse without a definite cause which was not identified despite a series of work up. However, with adjustment of exercise intensity to level of shortness of breath, the patient showed gradual improvement after continuous exercise therapy. The cause of severe shortness of breath might be due to the weakness of the inspiratory muscle, such as the external intercostal muscle, upper trapezius, and sternocleidomastoid. As he was progressing in his exercise, the patient was able to exercise more at the same level of dyspnea. It means that exercise therapy affected positively the exercise capacity, and it improved after 4 months although hospitalization was longer than in other previous cases. Thus a reduction in dyspnea may be responsible for improved exercise capacity in LVAD implantation. After LVAD implantation, a ventricular arrhythmia may develop in up to $50 \%$ of these patients [2] and is a significant indicator of the rise in the incidence of monomorphic ventricular tachycardia [8]. The patient in this case showed NSVT in a telemetry monitoring at POD 90. Sometimes, the patient felt an irregular rhythm during the exercises, such as bridge, straight leg raise, going up and down the stairs, and treadmill walking exercise. When the ventricular tachycardia was detected, we reduced exercise intensity and sometimes gave a rest time for a few minutes until the disappearance of the arrhythmia. After 15 days, the arrhythmia would disappear spontaneously. In our case, these conditions affected 
the length of stay in the hospital. Compared to previous studies and to the exercise protocol including Utah and Columbia NYC rehabilitation protocols, recovery rate in our case was delayed due to some complications. Older age has shown to be an independent predictor of death throughout the duration of LVAD therapy [9]. Therefore, when these patients are enrolled in exercise therapy program after implanted LVAD, it is important to consider medical complications and age for the optimal exercise therapy. We observed his hemodynamic response for 2 weeks to decide the discharge time from our hospital, and no problems were found in this period. At this point (POD 136), he was discharged from the hospital with advice for daily activity and exercise. The 1-year survival rate after implanted LVAD was reported to be in the range from $69 \%$ to $85 \%$. We also anticipated a survival rate of 1 year in our case when considering the clinical state and examination results. In conclusion, we have documented a case of a LVAD implantation as a destination therapy in a patient with end-stage heart failure, in which effective and safe in-patient cardiac rehabilitation with the improvement of exercise capacity QOL was achieved. Therefore, it is important for patients with LVAD implantation to participate in inpatient exercise therapy for the improvement of dyspnea and QOL.

\section{CONFLICT OF INTEREST}

No potential conflict of interest relevant to this article was reported.

\section{REFERENCES}

1. Go AS, Mozaffarian D, Roger VL, Benjamin EJ, Berry JD, Borden WB, et al. Heart disease and stroke statistics: 2013 update: a report from the American Heart Association. Circulation 2013;127:e6-e245.
2. Nissinoff J, Tian F, Therattil M, Salvarrey RM, Lee SW. Acute inpatient rehabilitation after left ventricular assist device implantation for congestive heart failure. PM R 2011;3:586-9.

3. Miller LW, Pagani FD, Russell SD, John R, Boyle AJ, Aaronson KD, et al. Use of a continuous-flow device in patients awaiting heart transplantation. N Engl J Med 2007;357:885-96.

4. Corra U, Pistono M, Mezzani A, Gnemmi M, Tarro Genta F, Caruso R, et al. Cardiovascular prevention and rehabilitation for patients with ventricular assist device from exercise therapy to long-term therapy. Part I: Exercise therapy. Monaldi Arch Chest Dis 2011;76:27-32.

5. Reedy JE, Swartz MT, Lohmann DP, Moroney DA, Vaca KJ, McBride LR, et al. The importance of patient mobility with ventricular assist device support. ASAIO J 1992;38:M151-3.

6. Hasin T, Topilsky Y, Kremers WK, Boilson BA, Schirger JA, Edwards BS, et al. Usefulness of the six-minute walk test after continuous axial flow left ventricular device implantation to predict survival. Am J Cardiol 2012;110:1322-8.

7. Grady KL, Meyer PM, Mattea A, Dressler D, Ormaza S, White-Williams $\mathrm{C}$, et al. Change in quality of life from before to after discharge following left ventricular assist device implantation. J Heart Lung Transplant 2003;22:322-33.

8. Ziv O, Dizon J, Thosani A, Naka Y, Magnano AR, Garan $\mathrm{H}$. Effects of left ventricular assist device therapy on ventricular arrhythmias. J Am Coll Cardiol 2005;45:1428-34.

9. Kirklin JK, Naftel DC, Kormos RL, Stevenson LW, Pagani FD, Miller MA, et al. Third INTERMACS Annual Report: the evolution of destination therapy in the United States. J Heart Lung Transplant 2011;30:115-23. 